\title{
High-dose IL-2 for metastatic renal cell carcinoma: can the first antitumor immunotherapy be reinvented?
}

\author{
"Investigation into the long-term effects of high-dose IL-2 may not \\ only allow for prediction of response to treatment in the future, \\ but also identify novel therapeutic targets for future enhanced \\ immunotherapies."
}

Keywords: immunotherapy • interleukin-2 • kidney cancer • metastatic renal cell carcinoma

Immunotherapy for the treatment of cancer holds the unique promise of durable, systemic antitumor responses capable of targeting malignant disease throughout the body. Just 8 years after the discovery of IL-2 [1], this promise was first fulfilled in a small clinical trial utilizing systemic high-dose IL-2 (HD IL-2) demonstrating a complete response in a metastatic melanoma patient who was cured of all malignant disease and has remained disease free for more than 29 years $[2,3]$. In this same trial, three out of three metastatic renal cell carcinoma (mRCC) patients showed partial regression of disease. The most encouraging benefit of HD IL-2 therapy then and now is the potential for complete elimination of malignant disease in a small subset of patients $(\sim 5-10 \%)[3-5]$.

While immunotherapeutic treatments for cancer continue to evolve, HD IL-2 protocols $(600,000 \mathrm{U} / \mathrm{kg} /$ dose intravenously every 8 h) have remained relatively unchanged since inception as the first US FDA approved immunotherapeutic agent for cancer in 1992. At that time, approval was based on seven Phase II clinical trials that demonstrated that HD IL-2 treatment alone induced complete responses in 5\% and partial responses in 9\% of mRCC patients [6]. In the two decades since this trial, the percentage of complete responders and partial responders has increased mod- estly $[4,5]$. Since the median overall survival of mRCC is only 14 months [7], HD IL-2 is still a viable option with the vast majority of complete responders remaining disease free 20 years after treatment [3].

The biological activity of IL-2 has been largely determined by in vitro and preclinical studies that, to this point, have not been translated into improvements in IL-2 clinical protocols. Under homeostatic conditions, activated CD4 T cells in secondary lymph nodes are the main producers of IL-2. IL-2 is also produced to a lesser extent by CD8 $\mathrm{T}$ cells, natural killer (NK) cells, NK T cells and dendritic cells $[3,8,9]$. T cells, NK cells and monocytes have IL-2 receptor components while tumor cells do not express IL-2 receptors $[3,10]$. This highlights the fact that the mechanism of action for HD IL-2 is not based on direct anticancer effects, but depends on immunemediated pathways. Signaling through IL-2 receptor subunits induces activation of JAK and phosphorylation of STAT, MAPK and PI3K [9]. IL-2 stimulation improves CD8 and CD4 $\mathrm{T}$ cell proliferation and survival but can also influence immunosuppressive $\mathrm{CD}^{+}$, FoxP3 ${ }^{+} \mathrm{T}$ regulatory cells $\left(\mathrm{T}_{\text {reg }}\right)$ that express CD25, which enhances IL-2 receptor affinity for IL-2 [9,11].

The overall effect of systemic delivery of IL-2 is complicated by IL-2 receptor expres-
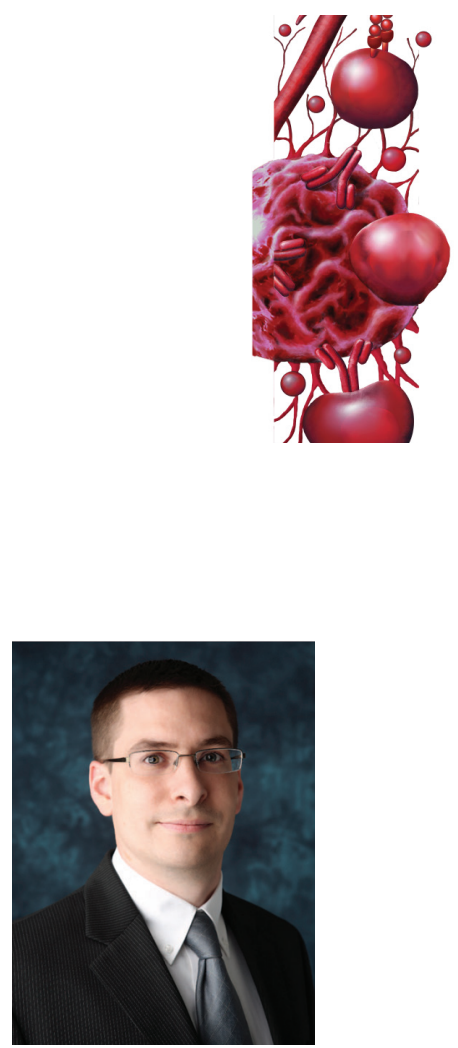

Jason B Muhitch Department of Urology, Roswell Park Cancer Institute, Buffalo, NY 14263, USA

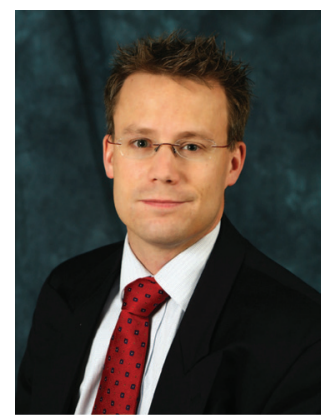

Thomas Schwaab Author for correspondence: Department of Urology, Roswell Park Cancer Institute, Buffalo, NY 14263, USA Tel.: + 17168458083 Fax: +17168453300 Thomas.Schwaab@ RoswellPark.org 
sion on both CD8 $\mathrm{T}$ cells that have the capacity to kill tumor cells and $\mathrm{T}_{\text {reg }}$ that can suppress CD8-mediated responses. Patients receiving HD IL-2 treatment have elevated levels of circulating $\mathrm{T}_{\mathrm{reg}}$ and this is correlated with poor prognosis [12-14]. Intratumoral $\mathrm{T}_{\mathrm{reg}}$ have also been shown to correlate with intratumoral CD8 $\mathrm{T}$ cells following IL-2 therapy suggesting that these cells can play an active role in dampening CD8 T-cell-mediated responses [13]. Indirect effects of IL-2 administration may also be partly responsible for side effects and clinical responses observed in patients. For instance, IL-2 can promote production of IL-6, IFN- $\gamma$, TNF- $\alpha$ and other cytokines in vitro [15]. Similarly, renal cell carcinoma (RCC) patients treated with IL-2 have elevated IFN- $\gamma$ and TNF- $\alpha$ levels during treatment when compared with levels following cessation of therapy [16]. IL-2 therefore not only impacts immune cell populations that can inhibit (cytotoxic CD8 $\mathrm{T}$ cells) and promote tumorigenesis (suppressive $\mathrm{T}_{\mathrm{reg}}$ ) but also initiates additional cytokine pathways such as IL-6 that can independently alter antitumor immunity.

The fact that even a small percentage of mRCC patients ( $\sim-10 \%)$ are essentially cured with HD IL-2 is astounding [5]. Clinical outcomes of HD IL-2 therapy strongly suggest that the immune system in complete responders is capable of eradicating bulky and disseminated malignant disease. Interestingly, durable complete responses to HD IL-2 rarely occur in other tumor types [17]; RCC, along with melanoma are the only malignancies documented to respond to HD IL-2 treatment. This and sporadic reports of spontaneous regression [18] have, in part, been responsible for the classification of melanoma and RCC as immunogenic tumors.

\footnotetext{
"The fact that even a small percentage of metastatic renal cell carcinoma patients $(\sim 5-10 \%)$ are essentially cured with high-dose IL-2 is astounding ... Interestingly, durable complete responses to high-dose IL-2 rarely occur in other tumor types."
}

Despite its curative potential, HD IL-2 remains under utilized in mRCC owing to its associated side effects and the relatively small number of patients who benefit from this treatment. The majority of HD IL-2 associated side effects are secondary to induction of autoimmune reactions. A major toxicity limiting the use of HD IL-2 is nephrotoxicity; however, multiple studies have now shown that HD IL-2 can safely be used for treatment of patients with mild renal dysfunction (serum creatinine level $\leq 1.9 \mathrm{mg} / \mathrm{dl}$ ). HD IL-2 is also limited by capillary leak syndrome that can impact end-organ function. Hypotension, elevated liver func- tion tests and signs of general systemic immune stimulation, such as chills and fever, are quite common [4]. Direct autoimmune cardiotoxic reactions in the form of myocarditis are rare [6]. Patient selection based on end-organ function, minimal comorbidities and performance status is important to minimize side effects. Patients presenting with clear cell RCC may also benefit more from HD IL-2 [19]. At the same time, this restricts the number of eligible patients. Approximately $2 \%$ of HD IL- 2 patients had treatment-related mortality in the first clinical trial more than 25 years ago. Since then, adjustments to patient selection and management of side effects have diminished patient-related mortality rates to $\leq 1 \%[3,4]$.

HD IL-2 therapy is hampered by the conundrum of how to determine which patients will respond to IL-2 treatment and how to alter therapy so even nonresponders will benefit from treatment. Even though HD IL-2 has been used for more than three decades, only incremental advances have been made in these areas. High intratumoral expression of CA9, a tumorassociated antigen (TAA), has been correlated with clinical responses to HD IL-2 in RCC [20]. This suggests that tumors expressing appropriate immune targets may respond better to therapy. Additionally, studies have shown that patients were least likely to respond if they had received other forms of therapy prior to HD IL-2 [4,21], supporting the use of HD IL-2 preferentially as first-line therapy for mRCC patients.

"...there is a dire need for biomarkers that reflect IL-2-mediated immunobiology ... Additionally, we need a toolkit of treatment strategies that utilize the pre-existing endogenous antigen-specific anticancer immunity in individual patients."

The ability of IL-2 to impact a broad range of immune cells implies that the status of a patient's endogenous immune system may predict responses to treatment. Early promising data from vaccines utilizing tumor-derived mRNA as a source of TAA support the hypothesis that immunogenic RCC-specific TAA may play a role in adaptive anti-RCC immunotherapy [22]. These data suggest that responding patients may have a population of TAA-specific CD8 T cells present that can be expanded by HD IL-2 therapy. In this context, we have reported on the use of dye dilution proliferation assays to interrogate tumor-specific $\mathrm{CD} 4$ and CD8 $\mathrm{T}$ cells [23]. The challenge is how to investigate these cell populations despite a paucity of information regarding individual TAAs in RCC. We and others are investigating the use of tetramers and dextramers of the available RCC-related TAAs, including CA9 and MUC-1, to facilitate this analysis. 
Clinical outcomes may also reflect failure to initiate immunosuppressive pathways. In a retrospective analysis of patients responding to HD IL-2 with autologous dendritic cell vaccination, partial or complete responders who had two cycles of therapy had statistically lower increases in circulating $\mathrm{T}_{\text {reg }}$ compared with nonresponding patients [14]. In this same clinical trial, gene profiling of circulating lymphocytes in responding patients revealed decreased immunosuppressive pathway activation following therapy [24]. Although tantalizing, these results only hint at the pretherapeutic immune state of responding patients. To date, there is a paucity of data available on the predictive role of T-cell populations prior to immunotherapy although definitive experiments are ongoing in our laboratory as well as others to identify IL-2-responsive T-cell signatures that can be correlated to clinical responses.

Future work evaluating HD IL-2 immunotherapy in RCC will have to focus on two major questions. Foremost, there is a dire need for biomarkers that reflect IL-2-mediated immunobiology. For this purpose, one might assess CD4 and CD8 versus $\mathrm{T}_{\text {reg }}$ cell populations to predict the likelihood of cytotoxic or suppressive responses resulting from IL-2 stimulation. Addition- ally, we need a toolkit of treatment strategies that utilize the pre-existing endogenous antigen-specific anticancer immunity in individual patients.

Above all, the most impressive finding is that this very nonspecific immunostimulatory molecule induces long lasting, possibly permanent immunity. We know that this cancer-specific immunity has to be mediated by memory $\mathrm{T}$ cells and other memory immune cell components; however, data on cancer-specific memory $\mathrm{T}$ cells in the context of HD IL-2 therapy are virtually nonexistent. Investigation into the long-term effects of HD IL-2 may not only allow for prediction of response to treatment in the future, but also identify novel therapeutic targets for future enhanced immunotherapies.

\section{Financial \& competing interests disclosure}

The authors have no relevant affiliations or financial involvement with any organization or entity with a financial interest in or financial conflict with the subject matter or materials discussed in the manuscript. This includes employment, consultancies, honoraria, stock ownership or options, expert testimony, grants or patents received or pending, or royalties.

No writing assistance was utilized in the production of this manuscript.

\section{References}

1 Morgan DA, Ruscetti FW, Gallo R. Selective in vitro growth of T lymphocytes from normal human bone marrows. Science 193(4257), 1007-1008 (1976).

2 Rosenberg SA, Lotze MT, Muul LM et al. Observations on the systemic administration of autologous lymphokineactivated killer cells and recombinant interleukin-2 to patients with metastatic cancer. N. Engl. J. Med. 313(23), 1485-1492 (1985).

3 Rosenberg SA. IL-2: the first effective immunotherapy for human cancer. J. Immunol. 192(12), 5451-5458 (2014).

4 Hanzly M, Aboumohamed A, Yarlagadda N et al. High-dose interleukin-2 therapy for metastatic renal cell carcinoma: a contemporary experience. Urology 83(5), 1129-1134 (2014).

5 Klapper JA, Downey SG, Smith FO et al. High-dose interleukin-2 for the treatment of metastatic renal cell carcinoma: a retrospective analysis of response and survival in patients treated in the surgery branch at the National Cancer Institute between 1986 and 2006. Cancer 113(2), 293-301 (2008).

6 Fyfe G, Fisher RI, Rosenberg SA, Sznol M, Parkinson DR, Louie AC. Results of treatment of 255 patients with metastatic renal cell carcinoma who received high-dose recombinant interleukin-2 therapy. J. Clin. Oncol. 13(3), 688-696 (1995).

7 Wood C, Srivastava P, Bukowski R et al. An adjuvant autologous therapeutic vaccine (HSPPC-96; vitespen) versus observation alone for patients at high risk of recurrence after 
IFN-\{alpha\}2a therapy in metastatic renal cell carcinoma patients. Clin. Cancer Res. 15(15), 4986-4992 (2009).

15 Economou JS, McBride WH, Essner R et al. Tumour necrosis factor production by IL-2-activated macrophages in vitro and in vivo. Immunology 67(4), 514-519 (1989).

16 Gemlo BT, Palladino MA Jr, Jaffe HS, Espevik TP, Rayner AA. Circulating cytokines in patients with metastatic cancer treated with recombinant interleukin 2 and lymphokine-activated killer cells. Cancer Res. 48(20), 5864-5867 (1988).

17 Dillman RO, Church C, Oldham RK, West WH, Schwartzberg L, Birch R. Inpatient continuous-infusion interleukin-2 in 788 patients with cancer. The National Biotherapy Study Group experience. Cancer 71(7), 2358-2370 (1993).

18 Middleton AW Jr. Indications for and results of nephrectomy for metastatic renal cell carcinoma. Urol. Clin. North Am. 7(3), 711-717 (1980).

19 Dandamudi UB, Ghebremichael M, Sosman JA et al. A Phase II study of bevacizumab and high-dose interleukin-2 in patients with metastatic renal cell carcinoma: a Cytokine Working Group (CWG) study. J. Immunother. 36(9), 490-495 (2013).
20 Atkins M, Regan M, McDermott D et al. Carbonic anhydrase IX expression predicts outcome of interleukin 2 therapy for renal cancer. Clin. Cancer Res. 11(10), 3714-3721 (2005).

21 Rosenberg SA, Yang JC, White DE, Steinberg SM.

Durability of complete responses in patients with metastatic cancer treated with high-dose interleukin-2: identification of the antigens mediating response. Ann. Surg. 228(3), 307-319 (1998).

22 Debenedette MA, Calderhead DM, Ketteringham $\mathrm{H}$ et al. Priming of a novel subset of $\mathrm{CD} 28^{+}$rapidly expanding high-avidity effector memory CTL by post maturation electroporation-CD40L dendritic cells is IL-12 dependent. J. Immunol. 181(8), 5296-5305 (2008).

23 Schwaab T, Ernstoff MS. Therapeutic vaccines in renal cell carcinoma. Therapy 4(8), 369-377 (2011).

24 Wolf B, Schwarzer A, Côté AL et al. Gene expression profile of peripheral blood lymphocytes from renal cell carcinoma patients treated with IL-2, interferon- $\alpha$ and dendritic cell vaccine. PLoS ONE 7(12), e50221 (2012). 